\title{
Identifying Appropriate Neuropsychological Tests for Uneducated/Illiterate Older Individuals
}

\author{
Maria-Aikaterini Mandyla ${ }^{1}$ (1) , Mary Yannakoulia ${ }^{2}$, Georgios Hadjigeorgiou ${ }^{3,4}$, Efthimios Dardiotis ${ }^{4}$, \\ Nikolaos Scarmeas ${ }^{5,6}$ and Mary H. Kosmidis ${ }^{1, *}$ \\ ${ }^{1}$ Lab of Cognitive Neuroscience, School of Psychology, Aristotle University of Thessaloniki, Thessaloniki, Greece \\ ${ }^{2}$ Department of Nutrition and Dietetics, Harokopio University, Athens, Greece \\ ${ }^{3}$ Department of Neurology, Medical School, University of Cyprus, Nicosia, Cyprus \\ ${ }^{4}$ Department of Neurology, Faculty of Medicine, University of Thessaly, Larissa, Greece \\ ${ }^{5}$ Department of Neurology, Aiginition Hospital, National and Kapodistrian University of Athens Medical School, Athens, Greece \\ ${ }^{6}$ Taub Institute for Research in Alzheimer's Disease and the Aging Brain, The Gertrude H. Sergievsky Center, Department of Neurology, \\ Columbia University, New York, NY, USA
}

(Received November 27, 2020; Final revision June 29, 2021; Accepted June 30, 2021; First Published Online August 31, 2021)

\begin{abstract}
Objectives: We investigated the utility of traditional neuropsychological tests in older uneducated/illiterate individuals without dementia to determine the possibility that they are likely not appropriate for this group. Methods: We assessed the neuropsychological performance of 1122 older adults [ $\geq 65$ years old; mean age: $74.03(S D=5.46)$; mean education: $4.76(S D=2.5)$ years; women: $n=714]$, in the context of the Hellenic Longitudinal Investigation of Aging and Diet (HELIAD), a population-based study conducted in Greece. Results: We based our analyses on three groups: highfunctioning/cognitively healthy (i.e., without dementia) uneducated/illiterate individuals $(n=80)$, high-functioning/ cognitively healthy educated/literate individuals $(n=932)$, and low-functioning/cognitively impaired educated/literate individuals (presumably with dementia; $n=110$ ). We used binary regression analyses with Bonferroni correction to investigate whether test performance differentiated uneducated/illiterate from educated/literate individuals. Models were adjusted for age and sex; raw test scores were the predictor variables. The uneducated/illiterate cohort was at a disadvantage relative to the healthy educated/literate group on all variables but verbal memory recognition and consolidation, congruent motor responses, and phonological fluency clustering $(p>.002)$. Moreover, only word list learning immediate and delayed free recall and delayed cued recall differentiated the high-functioning/cognitively healthy uneducated/illiterate from the low-functioning/cognitively impaired educated/literate group, favoring the former ( $p$ 's < .002). Conclusions: Our findings suggest that only particular verbal memory test variables are fair in determining whether older uneducated/illiterate individuals have functional/cognitive impairment suggestive of a neurodegenerative process. On all other neuropsychological variables, this cohort was at a disadvantage. Therefore, we highlight the need for identifying appropriate methods of assessment for older uneducated/illiterate individuals.
\end{abstract}

Keywords: Functional, Assessment, Diagnosis, Impairment, Aging, Dementia

\section{INTRODUCTION}

A notable proportion of the world's population is illiterate and/or lacks formal education. In many societies, mainly in industrialized countries, uneducated/illiterate individuals are over-represented among the elderly (UIS, eAtlas of Literacy, 2020). Given the frequency of cognitive decline with age, many uneducated/illiterate older individuals may at some point require a neuropsychological assessment.

*Correspondence and reprint requests to: Mary H. Kosmidis, PhD, Lab of Cognitive Neuroscience, School of Psychology, Aristotle University of Thessaloniki, 54124 Thessaloniki, Greece. E-mail: kosmidis@psy.auth.gr
As psychometric tests are typically developed for literate individuals with formal schooling experience, performance on them may be affected by factors such as culture, language, education, and literacy (Ardila et al., 2010; Nielsen \& Waldemar, 2016). Therefore, the use of such tests may lead to an underestimation of the cognitive abilities of uneducated/ illiterate individuals, potentially overestimating the probability of neuropathology. Consequently, it is imperative that appropriate psychometric tools for these populations are identified.

Many investigations of the neuropsychological correlates of illiteracy and/or lack of schooling have focused on 
performance differences between uneducated/illiterate and educated/literate individuals, documenting poorer performance among the former compared to the latter. Indeed, healthy uneducated/illiterate individuals tend to underperform on tests of language (Kosmidis, Tsapkini, \& Folia, 2006; Reis \& Castro-Caldas, 1997), with the exception of supermarket fluency (Reis, Guerreiro, \& Petersson, 2003) and verbal recognition (da Silva, Petersson, Faisca, Ingvar, \& Reis, 2004; Youn et al., 2011); visuospatial perception (Brucki \& Nitrini, 2008; Byrd, Jacobs, Hilton, Stern, \& Manly, 2005; Hong et al., 2011), with the exception of the Remembering-a-New-Route task (Kosmidis, Zafiri, \& Politimou, 2011) and object learning (Chung, 2009; Folia \& Kosmidis, 2003); memory and learning (Ardila, Rosselli, \& Rosas, 1989; Manly et al., 1999; Nitrini et al., 2004); attention (van Linden \& Cremers, 2008); working memory (Kosmidis et al., 2011); and executive functioning (Gómez, Zunzunegui, Lord, Alvarado, \& García, 2013). The format of these tests resembles that of tasks typically used in school.

Previous research has identified or developed tests in an attempt to overcome the limitations of most traditional measures for the neuropsychological assessment of uneducated/illiterate individuals by including items or procedures that reflect knowledge or activities typical of daily living rather than school-type tasks. Some such tests include as follows: the Brief Cognitive Screening Battery (Nitrini et al., 2004), Rivermead Behavioural Memory Test (Wilson, Cockburn, Baddeley, \& Hiorns, 1989; Yassuda et al., 2009), Informant Questionnaire for Cognitive Decline in the Elderly, Rowland Universal Dementia Screening (Araujo, Nielsen, Engedal, Barca, Coutinho, \& Laks, 2018; Goudsmit, van Campen, Franzen, van den Berg, Schilt, \& Schmand, 2020; Nielsen, Phung, Chaaya, Mackinnon, \& Waldemar, 2016), Persian test of Elderly for Assessment of Cognition and Executive Function (Javadi, Zendehbad, Darabi, Khosravifar, \& Noroozian, 2015), Hindi Mental State Examination (Ganguli et al., 1995; Tiwari, Tripathi, \& Kumar, 2009), Fuld Object Memory Evaluation (Chung, 2009), Recall of Pictures Test (naming, delayed recall, and recognition) (Nielsen, Vogel, \& Waldemar, 2012), Enhanced Cued Recall (Araujo et al., 2020), the TNI-93 (Maillet et al., 2016), and Cross-Cultural Dementia Screening (Goudsmit et al., 2017). Despite the development of these tests, however, many epidemiological and other studies of illiteracy, as well as clinical assessments, continue to utilize traditional, commonly used neuropsychological tests.

Several studies have suggested that the process of formal schooling and learning to read and write changes the way the brain functions (Reis \& Castro-Caldas, 1997; Reis et al., 2007). Specifically, the acquisition of reading and writing skills has been found to enhance fundamental cognitive abilities, such as memory processes, phonological awareness, visuospatial and visuomotor skills, attention, and executive functioning, in addition to language skills (for a review, see Ardila et al., 2010). To the extent that diagnostic procedures for identifying neurodegenerative processes include neuropsychological tests developed for assessing cognitive impairment relying on these specific skills, people who have not acquired them will be at a disadvantage relative to those who have, potentially giving the impression of cognitive impairment indicative of neuropathology in the former group.

Several studies have explored the similarities between older uneducated/illiterate, functionally illiterate, or low educated (with $\leq 6$ years of schooling) individuals and educated/ literate individuals with dementia (Kim \& Chey, 2010; Youn et al., 2011). These investigations have reported that commonly used neuropsychological tests [i.e., Clock Drawing Test (CDT), Mini-Mental State Exam (MMSE), Boston Naming Test, Rosen Drawing Test], do not differentiate healthy uneducated/illiterate individuals from educated/ literate individuals with Alzheimer's disease (AD) (Kim \& Chey, 2010; Youn et al., 2011). Indeed, qualitative analysis has shown similar conceptual errors on the CDT among illiterate individuals or those with low education and literate individuals with AD (Kim \& Chey, 2010; Nielsen \& Jørgensen, 2013). If tests such as the CDT fail to differentiate healthy illiterate or low education individuals from educated groups with dementia, their usefulness in a clinical assessment becomes questionable.

Consequently, many of the commonly used neuropsychological tests may not be reliable measures for the assessment of cognitive ability in uneducated/illiterate individuals, since they are artificial laboratory tasks that resemble skills acquired, and cognitive processes practiced, in school. Typically, neuropsychological assessment with school-type tasks relies on the inherent assumption that examinees have fully developed and practiced skills or abilities. Instead, in the case of uneducated/illiterate individuals who lack this training, we may actually be measuring factors extrinsic to the test itself and the construct which we intend to measure (Kosmidis, 2018; Nell, 2000). Moreover, research has shown qualitative differences in the cognitive strategies used when comparing educated/literate and uneducated/ illiterate individuals (Kosmidis et al., 2006, 2011; Kosmidis, Vlahou, Panagiotaki, \& Kiosseoglou, 2004; Lachmann, Khera, Srinivasan, \& van Leeuwen, 2012; Reis \& Castro-Caldas, 1997; Reis, Faisca, Mendoca, Ingvar, \& Petersson, 2007). Based on these findings, normative data stratified by level of education may have little to contribute when assessing the cognitive functioning of uneducated/ illiterate individuals. In addition, some tests such as the Trail Making Test-Part B and the CDT have a high rate of failure or denial (e.g., 100\% failure rate among healthy controls) among uneducated/illiterate cohorts (Kim, Baek, \& Kim, 2014; Salmon, Jin, Zhang, Grant, \& Yu, 1995, cf Franzen et al., 2020). Therefore, the development of normative data may seem meaningless when a task has been shown to be excessively difficult for, or unfamiliar to, uneducated/illiterate individuals. When normative data are developed, however, they should be separate from those developed for low education cohorts. In contrast, uneducated/ illiterate individuals may be as effective as educated/literate individuals in coping with tasks representing activities 
encountered in daily life, tasks in which they may, presumably, have sufficient previous experience to have automatized relevant skills (Kempler, Teng, Taussig, \& Dick, 2010; Reis, Petersson, Castro-Caldas, \& Ingvar, 2001). Such findings highlight the necessity to examine the utility of particular neuropsychological tests for use in the clinical diagnosis of uneducated/illiterate individuals, as well as to reduce or eliminate test bias in the assessment of this group.

Indeed, several tests specific to individuals with low levels of or no education have been developed in recent years (for a review, see Franzen et al., 2020), such as the International Shopping List Test (Thompson et al., 2011), the Multiple Errands Test (Alderman, Burgess, Knight, \& Henman, 2003), or its Virtual Reality (VR) version (Cipresso et al., 2014), or other VR tests, such as the Non-immersive Virtual Coffee Task (Besnard et al., 2016) or the Multitasking in the City Test (Jovanovski et al., 2012). Despite the progress that has been made in the field, the available evidence has shown that most tests have been inadequately validated to ensure a fair assessment, especially for a diagnosis based on a wide range of cognitive functions, in uneducated/illiterate populations (for a review, see Paddick et al., 2017) and traditional tests with inadequate norms are still often used both in research and in clinical practice.

Another issue of critical importance when comparing healthy older cohorts to those with cognitive decline indicative of neuropathology (e.g., dementia) is the inherent use of neuropsychological measures to support a potential diagnosis. Thus, when exploring the utility of particular tests for older uneducated/illiterate individuals, determining who is cognitively healthy and who is not, must not be based on the findings of a neuropsychological assessment. To avoid such circular reasoning, independent measures of functioning are needed to determine health status (some or no decline indicative of a neurodegenerative process).

In the present study, we explored neuropsychological test performance similarities and differences between healthy older uneducated/illiterate and educated/literate individuals, as well as between healthy older uneducated/illiterate individuals and their educated/literate counterparts with documented cognitive/functional impairment, to identify those tests that are not appropriate and those tests that are potentially useful tools in the assessment of uneducated/illiterate individuals without dementia.

\section{METHODS}

\section{Participants}

We assessed adults $\geq 65$ years old for the Hellenic Longitudinal Investigation of Aging and Diet (HELIAD), an epidemiologic, longitudinal, population-based study conducted in Greece. Table 1 lists the sample demographic characteristics. All participants were native speakers of Greek and communicated with the researchers in Modern Greek (the sole form used in schools, the media, and public and other services), despite any local dialects some may use at home; ethnicity was considered homogenous.

We divided our participants into four groups, firstly, based on their educational status (attended school or not), and then based on their cognitive/functional status (healthy or with cognitive/functional impairment). This yielded 80 uneducated/illiterate participants, 932 cognitively healthy (including only those with 1-6 years of education, so as to avoid a large difference with the no education group), and 110 cognitively impaired literate/educated individuals (education ranging from 1 to 18 years). The group of uneducated/illiterate participants with low-functioning/ cognitive impairment was too small $(n<10)$ to include in any analyses, therefore it will not be discussed further. We circumvented the use of clinical diagnoses such as mild cognitive impairment and dementia to determine health/ impairment status, as these were based (in our study, as is customary) both on neurological examination and a neuropsychological assessment with the same tests whose appropriateness we were investigating. Thus, to avoid circular reasoning, we did not distinguish our groups based on neuropsychological test scores (i.e., considering participants with low performance as having dementia and vice versa). Instead, we screened them for cognitive impairment using the criterion of their level of functioning relevant to dementia symptoms. Specifically, we considered high-functioning individuals as cognitively healthy and low-functioning individuals as having dementia/with cognitive impairment. Therefore, we defined this clinical criterion based on scales used worldwide for the assessment of dementia, rather than neuropsychological tests scores, as the latter were used as predictor variables. Thus, to evaluate the level of functioning, we used a combination of the Instrumental Activities of Daily Living (IADL-short version; Lawton \& Brody, 1969), the Blessed Dementia Rating Scale-Activities of Daily Living (BDRS; Blessed, Tomlinson, \& Roth, 1968), and the Clinical Dementia Rating (CDR; Morris, 1993).

We included the CDR as one of our criteria because it was the only scale of the three chosen that has defined cut-off scores to determine the adequacy of cognitive and functional abilities. All three scales were selected based on their widespread use and validity as measures for rating dementia through the assessment of cognitive domains and/or functional behavior changes in daily activities. We chose to use this combination of scales in order, as each focuses on a different aspect of functioning, yet all are relevant for our purposes. Table 2 lists the combination of cut-off scores we used for the determination of high-functional (presumably without dementia) versus low-functional status (presumably with dementia).

\section{Procedure}

Participants were selected through random sampling from municipality records. The consent form was read to uneducated/illiterate individuals, and explained, if needed; participants signed the form with a cross or their initials. 
Table 1. Demographic characteristics and other descriptive variables by group

\begin{tabular}{|c|c|c|c|c|}
\hline & $\begin{array}{l}\text { HF/CH Illiterate/ } \\
\text { Uneducated }(n=80)\end{array}$ & $\begin{array}{l}\text { HF/CH Literate/Educated } \\
\text { (1-6y) }(n=932)\end{array}$ & $\begin{array}{c}\text { LF/CI Literate/ } \\
\text { Educated }(n=110)\end{array}$ & $\begin{array}{l}\text { Total Sample } \\
\quad(n=1122)\end{array}$ \\
\hline Language (monolingual Greek) $[n(\%)]$ & $79(98.8)$ & 929 (99.7) & $110(100)$ & 1118 \\
\hline Other language & $1(1.3)$ & $2(.2)$ & & 3 \\
\hline Missing & & $1(.1)$ & & 1 \\
\hline \multicolumn{5}{|l|}{ Handedness $[n(\%)]$} \\
\hline Right handed & $77(96.3)$ & 875 (93.9) & $105(95.5)$ & 1057 \\
\hline Left handed & & $15(1.6)$ & $1(.9)$ & 16 \\
\hline Ambidextrous & $1(1.3)$ & $16(1.7)$ & $1(.9)$ & 18 \\
\hline Missing & $2(2.5)$ & $26(2.8)$ & $3(2.7)$ & 31 \\
\hline \multicolumn{5}{|l|}{$\operatorname{Sex}[n(\%)]$} \\
\hline Male & $15(18.8)$ & $346(37.1)$ & $47(42.7)$ & 408 \\
\hline Female & $65(81.2)$ & $586(62.9)$ & $63(57.3)$ & 714 \\
\hline \multirow[t]{2}{*}{ Age (SD) (y) } & $75.7(4.17)$ & $73.44(5.31)$ & $77.8(S D=5.8)$ & \\
\hline & {$[68-88]$} & [65-91] & [65-93] & \\
\hline \multirow[t]{2}{*}{ Education (SD) (y) } & $0(0)$ & $4.91(1.57)$ & $6.95(4.67)$ & \\
\hline & {$[0]$} & [1-6] & {$[1-18]$} & \\
\hline \multicolumn{5}{|l|}{ Occupation when working $[n(\%)]$} \\
\hline Homemaker & $30(37.5)$ & $338(36.3)$ & $27(24.5)$ & 395 \\
\hline Farmer & $22(27.5)$ & $152(16.3)$ & $14(12.7)$ & 188 \\
\hline Stockbreeder & $2(2.5)$ & $5(.5)$ & $2(1.8)$ & 9 \\
\hline Worker & $20(25)$ & $197(21.1)$ & $16(14.5)$ & 233 \\
\hline Craftsman & $1(1.3)$ & $78(8.4)$ & $14(12.7)$ & 93 \\
\hline Self-employed & $3(3.8)$ & $92(9.9)$ & $13(11.8)$ & 108 \\
\hline Civil servant in office & $1(1.3)$ & $13(1.4)$ & $3(2.7)$ & 17 \\
\hline Civil servant worker & $1(1.3)$ & $14(1.5)$ & $2(1.8)$ & 17 \\
\hline Work in private office & & $13(1.4)$ & $7(6.4)$ & 20 \\
\hline Teacher & & $1(.1)$ & $3(2.7)$ & 4 \\
\hline Business manager & & & $2(1.8)$ & 2 \\
\hline \multicolumn{5}{|l|}{ Unemployed } \\
\hline Other & & $29(3.1)$ & $6(5.5)$ & 35 \\
\hline Missing & & & $1(.9)$ & 1 \\
\hline \multicolumn{5}{|l|}{ Health Status } \\
\hline Number of clinical & $2.05(1.54)$ & $2.2(1.55)$ & $2.5(1.5)$ & \\
\hline Comorbidities & {$[0-7]$} & {$[0-9]$} & [0-6] & \\
\hline \multicolumn{5}{|l|}{ Marital Status $[n(\%)]$} \\
\hline Married & $50(62.5)$ & $673(72.2)$ & $77(70)$ & 800 \\
\hline Not married & $30(37.5)$ & $259(27.8)$ & $32(29.1)$ & 321 \\
\hline Missing & & & $1(.9)$ & 1 \\
\hline \multicolumn{5}{|l|}{ Region $[n(\%)]$} \\
\hline Larisa & $79(98.8)$ & 854 (91.6) & $83(75.5)$ & 1.016 \\
\hline Marousi & $1(1.2)$ & $78(8.4)$ & $27(24.5)$ & 106 \\
\hline \multicolumn{5}{|l|}{ MMSE items (28 and 29) $[n(\%)]$} \\
\hline Performed both & $10(12.5)$ & $781(83.8)$ & $63(57.3)$ & 854 \\
\hline Performed neither & $60(75)$ & $46(4.9)$ & $17(15.5)$ & 123 \\
\hline Performed one & $7(8.8)$ & $83(8.9)$ & $21(19.1)$ & 111 \\
\hline Missing & $3(3.8)$ & $22(2.4)$ & $9(8.2)$ & 34 \\
\hline
\end{tabular}

Abbreviations: HF, high functioning; $\mathrm{CH}$, cognitively healthy, LF, low functioning; CI, cognitively impaired; y, years; MMSE, Mini-Mental State Examination. Percentages for categorical variables indicate the relative frequency of the characteristic in the population of the respective column.

MMSE item 28: read/interpret the command "Close your eyes", MMSE item 29: write a sentence.

Educated/literate individuals read the form themselves and signed it after any questions were answered by the experimenter. The study protocol was approved by the University of Thessaly (UoT) and the National and Kapodistrian University of Athens ethics committees. The research was completed in accordance with the Helsinki Declaration. All received a comprehensive neurological and a neuropsychological evaluation in Greek leading to a consensus diagnosis by boardcertified neurologists and trained neuropsychologists who had previous experience working with older adults with a heterogeneous educational background. The functional criteria (IADL, BDRS, and CDR) were part of the procedure and 
Table 2. Criteria to determine functional status based on dementia rating and functional scales

\begin{tabular}{lcccccc}
\hline \hline \multicolumn{3}{c}{ High functional } & & \multicolumn{3}{c}{ Low functional } \\
\cline { 1 - 2 } \cline { 5 - 7 } CDR & BDRS & IADL & & CDR & BDRS & IADL \\
\hline 0 & - & - & & 1 & - & - \\
.5 & $\leq 3$ & - & & .5 & $\geq 4$ & - \\
.5 & - & $=4$ & & .5 & - & $\leq 3$ \\
.5 & $\leq 3$ & $=4$ & & .5 & $\geq 4$ & $\leq 3$ \\
- & $\leq 3$ & $=4$ & & - & $\geq 4$ & $\leq 3$ \\
\hline \hline
\end{tabular}

CDR, clinical dementia rating scale; BDRS, blessed dementia rating scale; IADL, instrument of activities of daily living.

considered an important factor for defining functional/cognitive status, thus were chosen as a reference standard in the present study. Sessions took place at day-care centers for the elderly, their homes, the UoT Medical Center, or municipal public health clinics. The recruitment process and neurological evaluation of participants have been described in full elsewhere (Dardiotis, Kosmidis, Yannakoulia, Hadjigeorgiou, $\&$ Scarmeas, 2014). All received the same battery of neuropsychological tests and in the same order (approximate duration $=1 \mathrm{hr}$ ). The battery consisted of tests that are widely used as standardized measures (in many countries, including Greece) of memory, visuospatial ability, attention/information processing speed, language, and executive functioning. In the present analyses, we included several process scores to discern whether potential group differences in total score may be reflected in different cognitive strategies, and vice versa, to discern whether potential group differences were quantitative and did not reflect differences in cognitive strategies.

\section{Neuropsychological evaluation}

The neuropsychological battery included the following tests:

1. Medical College of Georgia Complex Figure Test (MCGCF) (Lezak, Howieson, \& Loring, 2004). Variables of interest: copy, immediate and delayed recall of complex, abstract line drawing.

2. Greek Verbal Learning Test (GVLT) (Vlahou et al., 2013). Variables of interest: immediate and delayed free recall, immediate and delayed cued recall, and recognition. Based on the Item-Specific Deficit Approach (ISDA) (Wright et al., 2009) we also calculated three deficit indicators that were used as predictor variables: encoding, consolidation, and retrieval.

3. Verbal Fluency: semantic (objects; VFS) and phonological (“A"; VFP) (Kosmidis et al., 2004). Variables of interest: a total number of words and clusters for each condition separately.

4. Subtests of the Greek version of the Boston Diagnostic Aphasia Examination short form: Boston Naming Test (BNT)-short form and Complex Ideational Material Subtest (CIMS), to assess verbal comprehension and repetition of words and phrases (Tsapkini, Vlahou, \& Potagas, 2010). Variables of interest: total scores.
5. Trail Making Test-Part A (TMTA) (Vlahou \& Kosmidis, 2002). Variable of interest: completion time.

6. Anomalous Sentence Repetition Test (ASRT) (Lezak et al., 2004). Variable of interest: total score.

7. Graphical Sequence Test (GST) (Lezak et al., 2004). Variable of interest: total score.

8. Motor Programming-Parts A (congruent; MPA) \& B (incongruent; MPB) (Lezak et al., 2004). Variables of interest: total score for each part.

9. Judgment of Line Orientation (JLO, short form) (Benton, Varney, \& Hamsher, 1978). Variable of interest: total number correct.

\section{Statistical Analyses}

We used binary regression analyses adjusted for age (in years) and sex (dichotomous) to investigate whether test performance differentiates uneducated/illiterate from educated/literate individuals. The raw scores of the tests were used as the predictor variable. The criterion of significance was set at $\alpha=.002$ based on Bonferroni correction ( $p$-value divided by total number of variables: .05/24). The effect size was estimated by odds ratios (E.S. OR) in regressions after the numerical predictors (i.e., age, test scores) were standardized. Statistical analyses were performed using SPSS software version 23.0.

\section{RESULTS}

Our sample comprised 1122 participants (714 women). Their ages ranged from 65 to 93 [mean age: $74.03(S D=5.46)$ ] and educational level from 0 to 18 years of school [mean education: $4.76(S D=2.5)]$. Based on the grouping criteria (described in the Methods), one group comprised 80 highfunctioning/cognitively healthy uneducated/illiterate individuals [mean age: $75.7(S D=4.17)$ years, 65 women]. Another group included 932 high-functioning/cognitively healthy educated/literate individuals [mean age: $73.44(S D=5.31)$ years, 586 women] who had attended 1-6 years of school [mean education: $4.91(S D=1.57)$ years]. A final group consisted of 110 low-functioning/cognitive impaired educated/ literate individuals [mean age: $77.8(S D=5.8)$ years, mean education: $6.95(S D=4.67)$ years, 63 women] with formal schooling (from 1 to 18 years). Table 3 lists mean, standard deviations, and range of test scores by group.

\section{High-Functioning Uneducated/Illiterate and Educated/Literate Individuals}

Table 4 lists findings regarding variables that differentiated the high-functioning uneducated/illiterate from the highfunctioning educated/literate group, as well as those that did not. In all cases, the predictor variables which differentiated the two groups indicated a disadvantage of the high-functioning uneducated/illiterate group. All GVLT variables, with the exception of recognition memory and the consolidation deficit index score, differentiated between 
Table 3. Mean, standard deviation, and range on neuropsychological tests of educated/literate and uneducated/illiterate individuals

\begin{tabular}{|c|c|c|c|}
\hline Neuropsychological test variable & HFI & HFL & LFL \\
\hline \multicolumn{4}{|l|}{ GVLT } \\
\hline Immediate free recall $(\max =16)$ & $\begin{array}{c}5.88(3.27)^{* *} \\
\quad n=78 \\
\text { [range: } 0-12 \text { ] }\end{array}$ & $\begin{array}{c}7.70(3.3)^{*} \\
n=888 \\
\text { [range: } 0-16 \text { ] }\end{array}$ & $\begin{array}{c}3.36(3.51)^{* *} \\
\quad n=84 \\
\text { [range: } 0-14]\end{array}$ \\
\hline Immediate recall with cues $(\max =16)$ & $\begin{array}{c}8.58(2.68)^{*} \\
n=78 \\
\text { [range: } 3-14]\end{array}$ & $\begin{array}{c}10.32(2.85)^{*} \\
\quad n=888 \\
\text { [range: } 1-16 \text { ] }\end{array}$ & $\begin{array}{c}6.64(3.68) \\
n=84 \\
\text { [range: } 0-15\end{array}$ \\
\hline Delayed recall $(\max =16)$ & $\begin{array}{c}6.40(3.59) * * \\
n=77 \\
\text { [range: } 0-13 \text { ] }\end{array}$ & $\begin{array}{c}8.09(3.78)^{*} \\
\quad n=886 \\
\text { [range: } 0-16 \text { ] }\end{array}$ & $\begin{array}{c}3.18(3.78)^{* *} \\
\quad n=83 \\
\text { [range: } 0-16 \text { ] }\end{array}$ \\
\hline Delayed recall with cues $(\max =16)$ & $\begin{array}{c}8.75(2.81)^{* *} \\
n=77 \\
\text { [range: } 3-14]\end{array}$ & $\begin{array}{c}10.34(2.98)^{*} \\
\quad n=885 \\
\text { [range: } 0-16 \text { ] }\end{array}$ & $\begin{array}{c}6.16(3.77)^{* *} \\
\quad n=82 \\
\text { [range: } 0-16 \text { ] }\end{array}$ \\
\hline Recognition $(\max =16)$ & $\begin{array}{c}14.36(1.92) \\
\quad n=74 \\
\text { [range: } 8-16 \text { ] }\end{array}$ & $\begin{array}{c}14.1(2.11) \\
n=877 \\
\text { [range: } 0-16 \text { ] }\end{array}$ & $\begin{array}{c}12.56(3.74) \\
n=80 \\
\text { [range: } 0-16 \text { ] }\end{array}$ \\
\hline \multicolumn{4}{|l|}{ ISDA } \\
\hline Encoding deficit index & $\begin{array}{c}9.82(3.17)^{*} \\
\quad n=51 \\
\text { [range: } 2-15]\end{array}$ & $\begin{array}{c}7.55(3.09)^{*} \\
\quad n=776 \\
\text { [range: } 0-15 \text { ] }\end{array}$ & $\begin{array}{c}11.30(3.32) \\
n=76 \\
\text { [range: } 1-16 \text { ] }\end{array}$ \\
\hline Consolidation deficit index & $\begin{array}{c}.19(.13) \\
n=51 \\
\text { [range: } 0-.67]\end{array}$ & $\begin{array}{c}.16(.14) \\
n=776 \\
\text { [range: } 0-1.25]\end{array}$ & $\begin{array}{c}.36(.3) \\
n=76 \\
\text { [range: } 0-1.62 \text { ] }\end{array}$ \\
\hline Retrieval deficit index & $\begin{array}{c}.58(.23)^{*} \\
n=51 \\
\text { [range: .14-1] }\end{array}$ & $\begin{array}{c}.48(.19)^{*} \\
n=776 \\
\text { [range: } 0-1.75]\end{array}$ & $\begin{array}{c}.60(.29) \\
n=76 \\
\text { [range: } 0-1]\end{array}$ \\
\hline \multicolumn{4}{|l|}{ MCGCF } \\
\hline Copy $(\max =36)$ & $\begin{array}{c}26.22(6.98)^{*} \\
n=66 \\
\text { [range: } 2.5-36]\end{array}$ & $\begin{array}{c}31.92(5.35)^{*} \\
n=882 \\
\text { [range: } 1.5-36]\end{array}$ & $\begin{array}{c}25.91(9.44) \\
n=88 \\
\text { [range: } 3-36 \text { ] }\end{array}$ \\
\hline Immediate Recall $(\max =36)$ & $\begin{array}{c}8.04(5.89)^{*} \\
n=63 \\
\text { [range: } 0-32 \text { ] }\end{array}$ & $\begin{array}{c}13.35(6.97)^{*} \\
\quad n=874 \\
\text { [range: } 0-33 \text { ] }\end{array}$ & $\begin{array}{c}6.23(6.62) \\
n=81 \\
\text { [range: 0-29] }\end{array}$ \\
\hline Delayed Recall $(\max =36)$ & $\begin{array}{c}6.96(5.92)^{*} \\
n=63 \\
\text { [range: } 0-31 \text { ] }\end{array}$ & $\begin{array}{c}11.8(7.06)^{*} \\
\quad n=864 \\
\text { [range: } 0-35 \text { ] }\end{array}$ & $\begin{array}{c}4.44(5.91) \\
n=77 \\
\text { [range: } 0-27 \text { ] }\end{array}$ \\
\hline JLO Line pairs & $\begin{array}{c}11.09(4.06)^{*} \\
n=66 \\
\text { [range: } 0-10 \text { ] }\end{array}$ & $\begin{array}{c}5.08(2.43)^{*} \\
\quad n=874 \\
\text { [range: } 0-10 \text { ] }\end{array}$ & $\begin{array}{c}12.37(4.42) \\
n=71 \\
\text { [range: } 0-10]\end{array}$ \\
\hline TMT Part A & $\begin{array}{c}151.48(81.86)^{*} \\
n=60 \\
\text { [range: } 58-396 \text { ] }\end{array}$ & $\begin{array}{c}100.86(51.97)^{*} \\
n=859 \\
\text { [range: } 21-379]\end{array}$ & $\begin{array}{c}177.37(138.6) \\
n=70 \\
\text { [range: } 42-700 \text { ] }\end{array}$ \\
\hline Anomalous Sentence Repetition $(\max =14)$ & $\begin{array}{c}9.51(3.72)^{*} \\
n=39 \\
\text { [range: } 2-14 \text { ] }\end{array}$ & $\begin{array}{c}11.42(2.68)^{*} \\
\quad n=709 \\
\text { [range: } 0-14 \text { ] }\end{array}$ & $\begin{array}{c}9.12(3.95) \\
n=84 \\
\text { [range: } 0-14]\end{array}$ \\
\hline Graphical Sequence $(\max =6)$ & $\begin{array}{c}2.6(1.29)^{*} \\
n=35 \\
\text { [range: } 0-4.5 \text { ] }\end{array}$ & $\begin{array}{c}4.24(1.19)^{*} \\
n=682 \\
\text { [range: } 0-6 \text { ] }\end{array}$ & $\begin{array}{c}3.15(1.61) \\
n=76 \\
\text { [range: } 0-6 \text { ] }\end{array}$ \\
\hline \multicolumn{4}{|l|}{ Motor Programming } \\
\hline Condition A (congruent) $(\max =20)$ & $\begin{array}{c}17.74(1.97) \\
n=39 \\
\text { [range: } 14-20]\end{array}$ & $\begin{array}{c}18.91(1.68) \\
n=699 \\
\text { [range: } 9-20 \text { ] }\end{array}$ & $\begin{array}{c}17.27(4.28) \\
n=85 \\
\text { [range: } 0-20]\end{array}$ \\
\hline Condition $B$ (incongruent) $(\max =20)$ & $\begin{array}{c}13.18(5.95)^{*} \\
\quad n=39 \\
\text { [range: } 1-20]\end{array}$ & $\begin{array}{c}17.63(3.51)^{*} \\
\quad n=696 \\
\text { [range: } 0-20 \text { ] }\end{array}$ & $\begin{array}{c}11.50(7.47) \\
n=82 \\
\text { [range: } 0-20 \text { ] }\end{array}$ \\
\hline
\end{tabular}


Table 3. (Continued)

\begin{tabular}{|c|c|c|c|}
\hline Neuropsychological test variable & HFI & HFL & LFL \\
\hline \multicolumn{4}{|l|}{ BDAE } \\
\hline Naming $(\max =15)$ & $\begin{array}{c}7.16(2.12)^{*} \\
n=79 \\
\text { [range: } 3-12 \text { ] }\end{array}$ & $\begin{array}{c}9.47(2.35)^{*} \\
\quad n=908 \\
\text { [range: } 3-15]\end{array}$ & $\begin{array}{c}7.71(3.65) \\
n=99 \\
\text { [range: } 1-15]\end{array}$ \\
\hline \multicolumn{4}{|l|}{ CIMS } \\
\hline Comprehension $(\max =12)$ & $\begin{array}{c}9.08(1.99)^{*} \\
n=78 \\
\text { [range: } 2-12 \text { ] }\end{array}$ & $\begin{array}{c}10.40(1.63)^{*} \\
n=881 \\
\text { [range: 3-12] }\end{array}$ & $\begin{array}{c}8.79(2.33) \\
n=95 \\
\text { [range: } 2-12 \text { ] }\end{array}$ \\
\hline Word/sentence repetition $(\max =6)$ & $\begin{array}{c}3.47(1.38)^{*} \\
n=79 \\
\text { [range: } 0-6]\end{array}$ & $\begin{array}{c}4.24(1.34)^{*} \\
n=907 \\
\text { [range: } 0-6]\end{array}$ & $\begin{array}{c}3.53(1.58) \\
n=97 \\
\text { [range: 0-6] }\end{array}$ \\
\hline \multicolumn{4}{|l|}{ VERBAL FLUENCY } \\
\hline Semantic total words & $\begin{array}{c}7.21(4.76)^{*} \\
n=78 \\
\text { [range: } 2-22 \text { ] }\end{array}$ & $\begin{array}{c}14.43(4.73)^{*} \\
n=906 \\
\text { [range: } 0-31]\end{array}$ & $\begin{array}{c}5.40(4.59) \\
n=98 \\
\text { [range: } 0-24]\end{array}$ \\
\hline Clusters & $\begin{array}{c}1.55(1.04)^{*} \\
n=78 \\
\text { [range: } 0-4]\end{array}$ & $\begin{array}{c}2.20(1.17)^{*} \\
n=906 \\
\text { [range: } 0-6 \text { ] }\end{array}$ & $\begin{array}{c}1.37(1.17) \\
n=98 \\
\text { [range: } 0-5 \text { ] }\end{array}$ \\
\hline Phonological total words & $\begin{array}{c}3.44(2.65)^{*} \\
n=48 \\
\text { [range: } 0-11]\end{array}$ & $\begin{array}{c}5.31(3.24)^{*} \\
n=882 \\
\text { [range: } 0-17]\end{array}$ & $\begin{array}{c}4.02(3.46) \\
n=94 \\
\text { [range: } 0-14 \text { ] }\end{array}$ \\
\hline Clusters & $\begin{array}{c}.02(.14) \\
n=48 \\
\text { [range: } 0-1 \text { ] }\end{array}$ & $\begin{array}{c}.18(.42) \\
n=882 \\
\text { [range: } 0-2 \text { ] }\end{array}$ & $\begin{array}{c}.12(.32) \\
n=94 \\
\text { [range: } 0-1]\end{array}$ \\
\hline
\end{tabular}

HFI, high-functional illiterate; HFL, high-functional literate; LFL, low-functional literate; max, maximum score; GVLT, Greek verbal learning test; ISDA, Item-Specific Deficit Approach; MCGCF, medical college of Georgia complex figure test; JLO, judgment of line orientation; TMT, trail making test; BDAE, Boston diagnostic aphasia examination; CIMS, complex ideational material subtest. *HFI vs. HFL, $* *$ HFI vs. LFL, at $p<.002$.

the two groups. Similarly, the MCGCF variables copy, immediate recall, and delayed recall, as well as the JLO differentiated the educated/literate and uneducated/illiterate groups, as did the predictor variables TMTA, ASRT, GST, and MPB; only MPA did not differentiate the two groups. Finally, all variables relevant to verbal tasks differentiated between educated/literate and uneducated/illiterate individuals, with the exception of the number of clusters generated on VFP.

Specifically, a one-unit change in performance increased the odds of the participant being educated/literate on immediate free recall by $17.1 \%$ (E.S.OR $=1.805$ ), on immediate recall with cues by $25.2 \%$ (E.S. OR $=2.030$ ), on delayed recall by $11.9 \%$ (E.S. OR $=1.590)$, and on delayed recall with cues by $22.2 \%$ (E.S. OR $=1.930$ ). A one-unit change on performance decreased the odds of the participant being educated/literate on encoding by $23.6 \%\left(\right.$ E.S. $\left.._{\text {OR }}=.390\right)$ and on retrieval by $91.2 \%$ (E.S. OR $=.578$ ). Also, a one-unit change in performance increased the odds of the participant being educated/literate on MCGCF copy by $11.4 \%$ (E.S.OR $=$ $1.846)$, on immediate recall by $12.8 \%$ (E.S. $. \mathrm{OR}=2.635)$, on delayed recall by $10.5 \%$ (E.S.OR $=2.262$ ), on the JLO by $30.7 \%$ (E.S. OR $=1.959$ ), on ASRT by $20.5 \%$ (E.S. $. \mathrm{OR}=$ 1.664), on GST approximately by one time (E.S.. OR $=$ 3.059 ), on MPB by $18.5 \%$ (E.S. $\mathrm{OR}=2.001$ ), on naming by $50 \%$ (E.S. OR $=3.462$ ), on repetition of words and phrases by $43.5 \%$ (E.S.OR $=1.666$ ), on comprehension by $42 \%$
(E.S. ${ }_{\text {OR }}=1.832$ ), on semantic fluency for a total number of words by $16.7 \%$ (E.S.OR $=2.286$ ) and clusters by $69.4 \%$ (E.S.OR $=1.983$ ), and on phonological fluency for a total number of words by $23.7 \%$ (E.S.. OR $=2.583$ ). A one-unit change in completion time (sec) of the TMTA decreased the odds of the participant being educated/literate by $1 \%$ (E.S.. OR $=.549$ ).

In sum, the neuropsychological tests that did not show differences between the high-functioning educated/literate and the high-functioning uneducated/illiterate groups were GVLT recognition and the consolidation deficit index $(p$ 's > .002), MPA and number of clusters generated on VFP, whereas the remaining test variables explored in this study placed the uneducated/illiterate group at a disadvantage $(p$ 's $<.002)$.

\section{High-Functioning Uneducated/Illiterate and Low-Functioning Educated/Literate Individuals}

Table 5 lists findings regarding variables that differentiated the high-functioning uneducated/illiterate from the lowfunctioning educated/literate group, as well as those that did not. The GVLT immediate and delayed free recall, as well as delayed cued recall differentiated the low-functioning educated/literate group from high-functioning uneducated/ illiterate favoring the former, whereas immediate cued recall, recognition memory, and the consolidation, encoding and 
Table 4. Binary logistic regression analyses for prediction of group membership (high-functional literate vs. Illiterate) based on neuropsychological test performance (predictor variables) adjusted for age and sex

\begin{tabular}{|c|c|c|c|c|c|}
\hline & N (HFI/HFL) & $\beta( \pm S E)$ & $p$ & OR $(95 \% \mathrm{CI})$ & $R^{2}(N)$ \\
\hline \multicolumn{6}{|l|}{ GVLT } \\
\hline Immediate free recall & $966(78 / 888)$ & $.158(.038)$ & $<.001$ & $1.171(1.087-1.261)$ & .116 \\
\hline Immediate recall with cues & $966(78 / 888)$ & $.225(.045)$ & $<.001$ & $1.252(1.148-1.367)$ & .136 \\
\hline Delayed recall & $963(77 / 886)$ & $.113(.033)$ & .001 & 1.119 (1.049-1.194) & .100 \\
\hline Delayed recall with cues & $962(77 / 885)$ & $.201(.043)$ & $<.001$ & $1.222(1.123-1.330)$ & .124 \\
\hline Recognition & $951(74 / 877)$ & $-.058(.066)$ & .375 & $.944(.830-1.073)$ & .074 \\
\hline \multicolumn{6}{|l|}{ ISDA } \\
\hline Encoding deficit index & $827(51 / 776)$ & $-.269(.055)$ & $<.001$ & $.764(.686-.851)$ & .190 \\
\hline Consolidation deficit index & $827(51 / 776)$ & $-1.745(.924)$ & .059 & $.175(.029-1.067)$ & .117 \\
\hline Retrieval deficit index & $827(51 / 776)$ & $-2.426(.722)$ & .001 & $.088(.021-.364)$ & .142 \\
\hline \multicolumn{6}{|l|}{ MCGCF } \\
\hline Copy & $948(66 / 882)$ & $.108(.017)$ & $<.001$ & $1.114(1.077-1.152)$ & .143 \\
\hline Immediate Recall & $937(63 / 874)$ & $.120(.025)$ & $<.001$ & $1.128(1.074-1.183)$ & .116 \\
\hline Delayed Recall & $927(63 / 864)$ & $.099(.023)$ & $<.001$ & $1.105(1.056-1.155)$ & .098 \\
\hline JLO Line pairs & $940(66 / 874)$ & $.268(.059)$ & $<.001$ & $1.307(1.165-1.467)$ & .110 \\
\hline TMT Part A & $919(60 / 859)$ & $-.010(.002)$ & $<.001$ & $.990(.987-.994)$ & .102 \\
\hline Anomalous Sentence Repetition & $748(39 / 709)$ & $.186(.054)$ & $<.001$ & $1.205(1.085-1.338)$ & .193 \\
\hline Graphical Sequence & $717(35 / 682)$ & $.850(.143)$ & $<.001$ & $2.340(1.768-3.098)$ & .297 \\
\hline \multicolumn{6}{|l|}{ Motor Programming } \\
\hline Condition A (congruent) & $738(39 / 699)$ & $.198(.072)$ & .006 & $1.219(1.058-1.404)$ & .175 \\
\hline Condition B (incongruent) & $735(39 / 696)$ & $.170(.032)$ & $<.001$ & $1.185(1.113-1.262)$ & .248 \\
\hline \multicolumn{6}{|l|}{ BDAE } \\
\hline Naming & $987(79 / 908)$ & $.406(.060)$ & $<.001$ & $1.500(1.335-1.687)$ & .186 \\
\hline \multicolumn{6}{|l|}{ CIMS } \\
\hline Comprehension & $959(78 / 881)$ & $.351(.063)$ & $<.001$ & $1.420(1.255-1.607)$ & .142 \\
\hline Word/sentence repetition & $986(79 / 907)$ & $.361(.089)$ & $<.001$ & 1.435 (1.205-1.710) & .106 \\
\hline \multicolumn{6}{|l|}{ VERBAL FLUENCY } \\
\hline Semantic total words & $984(78 / 906)$ & $.155(.029)$ & $<.001$ & $1.167(1.102-1.236)$ & .135 \\
\hline Clusters & $984(78 / 906)$ & $.527(.120)$ & $<.001$ & $1.694(1.340-2.142)$ & .114 \\
\hline Phonological total words & $930(48 / 882)$ & $.212(.058)$ & $<.001$ & $1.237(1.103-1.387)$ & .113 \\
\hline Clusters & $930(48 / 882)$ & $2.205(1.008)$ & .029 & $9.069(1.258-65.397)$ & .098 \\
\hline
\end{tabular}

HFI, high-functional illiterates; HFL, high-functional literates; SE, standard error; OR, odds ratio; CI, confidence interval; GVLT, Greek verbal learning test; ISDA, Item-Specific Deficit Approach; MCGCF, medical college of Georgia complex figure test; JLO, judgment of line orientation; TMT, trail making test; BDAE, Boston diagnostic aphasia examination; CIMS, complex ideational material subtest; $\mathrm{N}$, Nagelkerke $R^{2}$.

retrieval deficit indices did not. Specifically, a one-unit change in performance decreased the odds of the participant being low-functioning educated/literate on immediate free recall by $17.6 \%($ E.S. OR $=.485)$, on delayed free recall by $18.5 \%($ E.S. OR $=.430)$ and on delayed recall with cues by $17.8 \%($ E.S. OR $=.525)$. On the MCGCF and the JLO, none of the variables examined differentiated between the highfunctioning uneducated/illiterate and the low-functioning educated/literate groups. Additionally, TMTA, ASRT, GST, MPA, and MPB variables did not differentiate between the high-functioning uneducated/illiterate and the low-functioning educated/literate group. Finally, the BNT, the CIMS comprehension and repetition of words and phrases, VFS and VFP did not differentiate between the high-functioning uneducated/illiterate and the low-functioning educated/ literate group. Thus, the high-functioning uneducated/ illiterate group was indistinguishable from the low-functioning educated/literate group on all but a few verbal memory variables.

\section{DISCUSSION}

We investigated whether high-functioning/cognitively healthy older uneducated/illiterate individuals are at a disadvantage, when assessed with conventional neuropsychological tests, relative to their educated/literate counterparts, and whether their performance may be indistinguishable from that of low-functioning/cognitively impaired older educated/ literate individuals. We found that the high-functioning/ cognitively healthy uneducated/illiterate group performed more poorly than their high-functioning/cognitively healthy educated/literate peers on most tests. The only neuropsychological test variables which may be considered "fair" to them are word list recognition memory and the consolidation deficit index, calculated from scores on the same test. Similarly, we found that the high-functioning/cognitively healthy uneducated/illiterate group was indistinguishable from the low-functioning/cognitively impaired educated/ literate group on all test variables, except immediate and delayed free recall, and delayed cued recall on a verbal 
Table 5. Binary logistic regression analyses for prediction of group membership (high-functional illiterate vs. low-functional literate) based on neuropsychological test performance (predictor variables) adjusted for age and sex

\begin{tabular}{|c|c|c|c|c|c|}
\hline & $\mathrm{N}(\mathrm{HFI} / \mathrm{LFL})$ & $\beta( \pm S E)$ & $p$ & OR $(95 \% \mathrm{CI})$ & $\mathrm{R}^{2}(\mathrm{~N})$ \\
\hline \multicolumn{6}{|l|}{ GVLT } \\
\hline Immediate free recall & $162(78 / 84)$ & $-.193(.053)$ & $<.001$ & $.824(.744-.914)$ & .224 \\
\hline Immediate recall with cues & $162(78 / 84)$ & $-.153(.054)$ & .005 & $.858(.772-.955)$ & .178 \\
\hline Delayed free recall & $160(77 / 83)$ & $-.205(.049)$ & $<.001$ & $.815(.740-.897)$ & .253 \\
\hline Delayed recall with cues & $159(77 / 82)$ & $-.197(.055)$ & $<.001$ & $.822(.738-914)$ & .214 \\
\hline Recognition & $154(74 / 80)$ & $-.221(.080)$ & .005 & $.802(.686-937)$ & .182 \\
\hline \multicolumn{6}{|l|}{ ISDA } \\
\hline Encoding & $127(51 / 76)$ & $.118(.062)$ & .057 & $1.125(.997-1.270)$ & .245 \\
\hline Consolidation & $127(51 / 76)$ & $3.160(1.155)$ & .006 & $23.570(2.449-226.820)$ & .293 \\
\hline Retrieval & $127(51 / 76)$ & $.071(.768)$ & .927 & $1.073(.238-4.833)$ & .212 \\
\hline \multicolumn{6}{|l|}{ MCGCF } \\
\hline Copy & $154(66 / 88)$ & $-.005(.020)$ & .795 & $.995(.956-1.035)$ & .098 \\
\hline Immediate recall & $144(63 / 81)$ & $-.042(.029)$ & .143 & $.959(.907-1.014)$ & .124 \\
\hline Delayed recall & $140(63 / 77)$ & $-.077(.032)$ & .015 & $.926(.870-.985)$ & .131 \\
\hline JLO & $137(66 / 71)$ & $.114(.081)$ & .160 & $1.120(.956-1.313)$ & .091 \\
\hline TMT Part A & $130(60 / 70)$ & $.003(.002)$ & .120 & $1.003(.999-1.006)$ & .119 \\
\hline Anomalous Sentence Repetition & $123(39 / 84)$ & $.026(.061)$ & .674 & $1.026(.910-1.156)$ & .280 \\
\hline Graphical Sequence & $111(35 / 76)$ & $.278(.160)$ & .083 & $1.320(.965-1.807)$ & .304 \\
\hline \multicolumn{6}{|l|}{ Motor Programming } \\
\hline Condition A (congruent) & $124(39 / 85)$ & $-.054(.075)$ & .475 & $.948(.818-1.098)$ & .275 \\
\hline Condition B (incongruent) & $121(39 / 82)$ & $-.034(.033)$ & .297 & $.966(.906-1.031)$ & .275 \\
\hline \multicolumn{6}{|l|}{ BDAE } \\
\hline Naming & $178(79 / 99)$ & $.047(.054)$ & .388 & $1.048(.942-1.166)$ & .118 \\
\hline \multicolumn{6}{|l|}{ CIMS } \\
\hline Comprehension & $173(78 / 95)$ & $-.052(.076)$ & .494 & $.950(.819-1.101)$ & .116 \\
\hline Word/sentence repetition & $176(79 / 97)$ & $-.007(.110)$ & .947 & $.993(.800-1.231)$ & .115 \\
\hline \multicolumn{6}{|l|}{ VERBAL FLUENCY } \\
\hline Semantic total words & $176(78 / 98)$ & $-.085(.034)$ & .013 & $.919(.859-.982)$ & .162 \\
\hline Clusters & $176(78 / 98)$ & $-.065(.145)$ & .651 & $.937(.706-1.244)$ & .118 \\
\hline Phonological total words & $142(48 / 94)$ & $.039(.063)$ & .538 & $1.039(.919-1.176)$ & .146 \\
\hline Clusters & $142(48 / 94)$ & $2.274(1.084)$ & .036 & $9.716(1.161-81.303)$ & .204 \\
\hline
\end{tabular}

HFI, high-functional illiterate; LFL, low-functional literate; SE, standard error; OR, odds ratio, CI, confidence interval; GVLT, Greek verbal learning test; ISDA, Item-Specific Deficit Approach; MCGCF, medical college of Georgia complex figure test; JLO, judgment of line orientation; TMT, trail making test; BDAE, Boston diagnostic aphasia examination; CIMS, complex ideational material subtest; N, Nagelkerke $R^{2}$.

learning test, favoring the former. Thus, the use of variables other than verbal memory may lead to an underestimation of the cognitive abilities of high-functioning/cognitively healthy uneducated/illiterate individuals.

The fact that verbal recognition memory and the consolidation deficit index do not put the uneducated/illiterate group at a disadvantage relative to the educated/literate group, as opposed to recall, encoding, and retrieval deficit indices, may reflect difficulties of the former group specific to retrieval, as well as the use of different neural substrates and learning strategies employed by uneducated/illiterates and educated/literates. Through attaining literacy in a school context, individuals are trained to identify written linguistic symbols and to dissect language into its component parts, developing both semantic and phonological cognitive strategies which may enhance their performance on school-based cognitive tasks. In contrast, lacking this training, uneducated/ illiterate individuals tend to rely on semantic properties to process linguistic information, consequently performing poorly on verbal memory tasks (Kosmidis, 2018). While this lack of a group difference on verbal recognition memory may reflect a ceiling effect, suggesting that recognition memory is not a very sensitive measure, in the present study neither the high-functioning uneducated/illiterate nor the high-functioning educated/literate group showed such an effect.

The present findings highlight two important factors in appreciating the optimal methods for the neuropsychological assessment of uneducated/illiterate individuals. Firstly, literacy attainment affects the development of cognitive skills beyond mere reading and use of a pencil to write and/or draw. Subsequently, though functional in their daily activities, the present group of older uneducated/illiterate individuals was at a clear disadvantage relative to a functional educated/literate cohort with a low level of education, on verbal learning strategies, retrieval, and encoding, as well as other verbal skills, namely, confrontation naming of objects in the form of two-dimensional sketches, comprehension, and repetition. Also, they did poorly on measures of visual memory and visuospatial perception based on two-dimensional lines and figures (some requiring drawing), as well as attention/speed 
of information processing (assessed with a paper-and-pencil test) and executive functioning tasks. Specifically, this group produced fewer words on verbal fluency and was less successful than their educated/literate counterparts in switching set and in inhibiting an automatized response on both oral and motor tasks.

Secondly, we identified neuropsychological tests that are appropriate for uneducated/illiterate individuals. Our results both replicate and extend previous findings. Specifically, as verbal memory recognition and consolidation did not differentiate between the healthy uneducated/illiterate and healthy educated/literate individuals, these variables may be useful when assessing potential cognitive impairment among uneducated/illiterate individuals. Additionally, verbal immediate and delayed free recall, as well as delayed cued recall appear to be useful predictors to distinguish between the high-functioning/cognitively healthy uneducated/illiterate and the low-functioning/cognitively impaired educated/literate group; thus, these variables may also be considered useful. This finding may reflect the impairment most likely to occur, and most prominent, in the most common cause of cognitive impairment among the elderly, namely, dementia, which usually manifests early on as memory decline, leading educated/literate individuals with cognitive impairment to perform even more poorly than their healthy uneducated/illiterate peers on such tasks. Thus, these variables would be most appropriate for differentiating the latter group from the former. In contrast, on most other neuropsychological variables, the high-functioning/cognitively healthy uneducated/illiterate group was at a disadvantage relative to the high-functioning/ cognitively healthy educated/literate, and indistinguishable from the low-functioning/cognitively impaired educated/ literate, groups. Consequently, the use of such variables would not aid in interpreting the poor test performance of illiterate/ uneducated individuals without running the risk of misclassifying the uneducated/illiterate patient as being cognitively impaired. Of course, we are not advocating relying solely on verbal memory tests to make diagnostic decisions but are highlighting the overall pattern of performance one might expect in determining whether or not a patient presents clinically relevant cognitive decline.

Our results are consonant with previous findings regarding verbal learning, where verbal recognition memory did not differentiate between a healthy educated/literate and a healthy uneducated/illiterate group (Kang et al., 2015), as well as with studies reporting a disadvantage of healthy uneducated/ illiterate individuals on visuospatial tasks (for a brief review, see Ardila et al., 2010), most verbal tasks (Ardila, OstroskySolis, \& Mendoza, 2000; Ostrosky-Solis, Ardila, \& Rosselli, 1999), and tests of executive functioning and attention/ information processing speed (Landgraf, Beyer, Schaadt, \& van der Meer, 2011). Thus, the acquisition of reading and writing skills through formal schooling appears to affect cognitive processing and performance in most domains and this must be considered when assessing uneducated/illiterate individuals for cognitive impairment through a traditional neuropsychological assessment.
The present study has several potential limitations related to the generalizability of the findings. Language and cultural factors may be specific to our sample, rendering our findings non-applicable to other uneducated/illiterate individuals, as they are not a homogenous group. Illiterate and low educated individuals in the present study comprise a unique sample due to cultural, linguistic, geographical, social, economic, and personal factors. Specifically, in Greece, differences in educational attainment in this cohort (i.e., illiteracy, low or high literacy level) might be attributed to gender inequality (i.e., in the mid-20th century, education was considered redundant for girls but not boys, since the former were expected to run a household, raise children and manage agrarian responsibilities, thus, they may have been discouraged from attending school or urged to drop out); timing of the war (i.e., participants in the present study were at different ages and educational levels during World War II); social imperative of children to work; poverty, births, deaths, or diseases in a family; regional differences such as residing in a rural versus an urban area (i.e., rates of illiteracy in the present study were higher among rural residents); parental educational level (i.e., illiterate parents were less likely to send their children to school); and father's occupation (i.e., children whose father's occupation was not manual were more likely to be educated). The aforementioned cultural, language, and historical context may have imbued the present sample with unique characteristics, thus, we cannot presume the extent to which observed performance differences and similarities would match those among older adults in other countries.

Above and beyond the quantity of education is the issue of its quality. Thus, another potential limitation is that we cannot determine the quality and the consistency of any formal schooling received by many of the participants in the present sample due to social factors (i.e., World War II, civil war) during their elementary school years, as schools often closed for indefinite periods of time, not to mention the potential effects of war- and famine-related stress during early childhood development in this cohort. Some factors that may reflect the quality of formal education in Greece at the time our sample attended school are variability in student-teacher ratios; limited or no funding and access to textbooks; mixed grade levels in the same classroom; education outside of school setting at home or at church; partial absence from school due to work or family demands; pedagogical methods; qualification of the teachers (sometimes priests); value placed on personal achievement and academic success. Though these factors may be important aspects of education (Crowe et al., 2013), they were not assessed in the present study.

Furthermore, our methods of categorizing participants based on functioning and education may also limit the generalizability of our findings. In the former case, there are no standardized cut-off scores for the BDRS and IADLS. Thus, categorization of our sample as high or low functioning was based on the current literature related to the interpretation of values on these two scales. With respect to the use of 
schooling to divide participants into illiterate and literate groups, some of those who went to school may be functionally illiterate and some of those who never went to school may have learned to read and write later in life. In the latter case, we speculated that, despite having learned to read and write later in life, they had not had the opportunity to practice or acquire cognitive skills at the same level as those who had received formal schooling in childhood. No or limited schooling/illiteracy in childhood, especially in Western countries, may be associated with low-socioeconomic status and limited employment opportunities in adult life, by extension exposing one to few opportunities to enhance cognitive abilities. Moreover, studies have suggested that learning to read and write during childhood influences the functional organization of the adult brain (Castro-Caldas, Petersson, Reis, StoneElander \& Ingvar, 1998), probably in a different way than when learning these skills as an adult. If our sample inadvertently included any functionally illiterate individuals, their inclusion in the literate group would have decreased the mean neuropsychological performance of this group. Yet most neuropsychological test variables differentiated between the healthy educated/literate and uneducated/ illiterate individuals, so we believe that their effect, if any, was undetectable.

Despite some caveats, the present study has several strengths. To our knowledge, it is the first study in Greece to include a comprehensive neuropsychological evaluation of older individuals, thus yielding the largest illiterate sample in any studies conducted in Greece to date. Also, our sampling procedure yielded a representative sample of the aging population in Greece, including uneducated/illiterate individuals of both sexes (many relevant studies have focused only on women). Moreover, all participants were fully evaluated by experts (neuropsychologists and neurologists) and cognitive status was based on widely accepted criteria. Furthermore, we identified participants in our sample who had cognitive impairment through the use of scales of daily functioning, rather than a neuropsychological test battery, as is customary. Although the latter is typically used to determine cognitive impairment and support a potential diagnosis of a neurodegenerative process such as dementia, along with a comprehensive neurological workup, in the present study neuropsychological test scores were used as predictor variables. Thus, we avoided circularity in our logic and our analyses. Finally, we investigated performance on numerous neuropsychological tests, assessing major cognitive domains (namely, memory, visuospatial ability, attention/information processing speed, language skills, and executive functioning) and identified potentially useful tests - as well as those that may be inappropriate for the assessment of cognitive functioning in uneducated/ illiterate individuals.

Thus, the present study extends the current literature regarding the utility of neuropsychological tests in the clinical assessment of older uneducated/illiterate adults. We highlight the impact of education/literacy skills on neuropsychological test performance - including tests not requiring reading and writing - and we pose serious questions relevant to the appropriateness of traditional neuropsychological tests for the assessment of dementia in uneducated/illiterate individuals. Also, we underline the importance of developing normative data for uneducated/illiterate older individuals for those tests that are appropriate. These normative data should be specific to those with no education and separate from those with low levels of education. Hence, the present study challenges the validity of the clinical assessment of uneducated/illiterate individuals. It also raises the question whether the high prevalence of dementia in older uneducated/illiterate individuals that is often found in population-based epidemiological studies reflects actual deficits, or is an artifact resulting from the inappropriateness of these tests for this population, thus leading to false-positive results on cognitive screening tests which are used as a reference standard for a dementia diagnosis (Bich et al., 2019; Goudsmit et al., 2020). Finally, our approach may provide a model for researchers in other cultural contexts to explore relevant factors for the neuropsychological assessment of uneducated/illiterate older individuals in their own countries.

\section{CONCLUSIONS}

In conclusion, the present findings highlight the need to assess the appropriateness of many widely used neuropsychological tests for the assessment of uneducated/illiterate older individuals and/or to develop separate normative data for this group specifically. Our data show that the potential effect of education/literacy on cognitive abilities and the nature of the tasks may lead to an underestimation of the cognitive functioning of healthy older uneducated/illiterate individuals. Instead, clinical assessment of uneducated/ illiterate individuals should entail sensitive criteria derived from tasks relevant to daily activities (Ortega, Aprahamian, Borges, Cação, \& Yassuda, 2019), on which education/ literacy level has little or no effect, as well as neuropsychological tests that have demonstrated validity for this population and specific normative data.

With increasing longevity and the concomitant increase in cases of neurocognitive decline related to dementia, further research in this area is needed to improve clinical assessment for uneducated/illiterate individuals. Although we undertook the present study in the hopes that our findings could guide clinical procedures for the neuropsychological assessment of older uneducated/illiterate individuals in Greece, increasing movements of immigrant or refugee populations with varied literacy backgrounds to Western countries due to war and economic factors in other parts of the world make the present study increasingly relevant even for industrialized countries with very low rates of illiteracy. Thus, the present findings could be useful as a springboard for future studies in our own, as well as in other cultures and languages regarding the most appropriate methods and tasks for the accurate assessment of potential cognitive decline in older uneducated/ illiterate adults. 


\section{FINANCIAL SUPPORT}

This study was supported by the following grants: IIRG-09133014 from the Alzheimer's Association; 189 10276/8/9/ 2011 from the ESPA-EU program Excellence Grant (ARISTEIA), which is co-funded by the European Social Fund and Greek National resources, and DY2b/oik. 51657/14.4.2009 from the Ministry for Health and Social Solidarity (Greece).

\section{CONFLICTS OF INTEREST}

The authors declare no conflicts of interest.

\section{REFERENCES}

Alderman, N., Burgess, P.W., Knight, C., \& Henman, C. (2003). Ecological validity of a simplified version of the multiple errands shopping test. Journal of the International Neuropsychological Society: JINS, 9, 31-44. doi: 10.1017/S1355617703910046

Araujo, N.B., Nielsen, T.R., Barca, M.L., Engedal, K., Marinho, V., Deslandes, A.C., Coutinho, E.S., \& Laks, J. (2020). Brazilian version of the European Cross-Cultural Neuropsychological Test Battery (CNTB-BR): diagnostic accuracy across schooling levels. Brazilian Journal of Psychiatry, 42, 286-294. doi: 10.1590/1516-4446-2019-0539

Araujo, N.B., Nielsen, T.R., Engedal, K., Barca, M.L., Coutinho, E.S., \& Laks, J. (2018). Diagnosing dementia in lower educated older persons: validation of a Brazilian Portuguese version of the Rowland Universal Dementia Assessment Scale (RUDAS). Brazilian Journal of Psychiatry, 40, 264-269. doi: 10.1590/1516-4446-2017-2284

Ardila, A., Bertolucci, P.H., Braga, L.W., Castro-Caldas, A., Judd, T., Kosmidis, M.H., ... Rosselli, M. (2010). Illiteracy: the neuropsychology of cognition without reading. Archives of Clinical Neuropsychology, 25, 689-712. doi: 10.1093/arclin/ acq079

Ardila, A., Ostrosky-Solis, F., \& Mendoza, V.U. (2000). Learning to read is much more than learning to read: a neuropsychologically based reading program. Journal of the International Neuropsychological Society, 6, 789-801.

Ardila, A., Rosselli, M., \& Rosas, P. (1989). Neuropsychological assessment in illiterates: visuospatial and memory abilities. Brain and Cognition, 11, 147-166.

Benton, A.L., Varney, N.R., \& Hamsher, K.D. (1978). Visuospatial judgment. A clinical test. Archives of Neurology, 35, 364-367. doi: 10.1001/archneur.1978.00500300038006

Besnard, J., Richard, P., Banville, F., Nolin, P., Aubin, G., Le Gall, D., \& Allain, P. (2016). Virtual reality and neuropsychological assessment: The reliability of a virtual kitchen to assess daily-life activities in victims of traumatic brain injury. Applied Neuropsychology-Adult, 23, 223-235. doi: 10.1080/23279095. 2015.1048514

Bich, N.N., Dung, N.T.T., Vu, T., Quy, L.T., Tuan, N.A., Binh, N.T.T., ... Anh, L.V. (2019). Dementia and associated factors among the elderly in Vietnam: a cross-sectional study. International Journal of Mental Health Systems, 13. doi: 10. 1186/s13033-019-0314-7

Blessed, G., Tomlinson, B.E., \& Roth, M. (1968). The association between quantitative measures of dementia and of senile change in the cerebral grey matter of elderly subjects. The British Journal of Psychiatry : The Journal of Mental Science, 114, 797-811.

Brucki, S.M.D. \& Nitrini, R. (2008). Cancellation task in very low educated people. Archives of Clinical Neuropsychology, 23, 139-147. doi: 10.1016/j.acn.2007.11.003

Byrd, D.A., Jacobs, D.M., Hilton, H.J., Stern, Y., \& Manly, J.J. (2005). Sources of errors on visuoperceptual tasks: role of education, literacy, and search strategy. Brain and Cognition, 58, 251-257. doi: 10.1016/j.bandc.2004.12.003

Castro-Caldas, A., Petersson, K.M., Reis, A., Stone-Elander, S., Ingvar, M. (1998). The illiterate brain. Learning to read and write during childhood influences the functional organization of the adult brain. Brain, 121, 1053-1063. doi: 10.1093/brain/121.6. 1053

Chung, J.C.C. (2009). Clinical validity of Fuld object memory evaluation to screen for dementia in a Chinese society. International Journal of Geriatric Psychiatry, 24, 156-162. doi: 10.1002/gps.2085

Cipresso, P., Albani, G., Serino, S., Pedroli, E., Pallavicini, F., Mauro, A., \& Riva, G. (2014). Virtual multiple errands test (VMET): a virtual reality-based tool to detect early executive functions deficit in Parkinson's disease. Frontiers in Behavioral Neuroscience, 8, 405. doi: 10.3389/fnbeh.2014.00405

Crowe, M., Clay, O.J., Martin, R.C., Howard, V.J., Wadley, V.G., Sawyer, P., \& Allman, R.M. (2013). Indicators of childhood quality of education in relation to cognitive function in older adulthood. The Journals of Gerontology. Series A, Biological Sciences and Medical Sciences, 68, 198-204. doi: 10.1093/ gerona/gls 122

Dardiotis, E., Kosmidis, M.H., Yannakoulia, M., Hadjigeorgiou, G.M., Scarmeas, N. (2014). The Hellenic Longitudinal Investigation of Aging and Diet (HELIAD): rationale, study design, and cohort description. Neuroepidemiology, 43, 9-14. doi: $10.1159 / 000362723$

da Silva, C.G., Petersson, K.M., Faisca, L., Ingvar, M., \& Reis, A. (2004). The effects of literacy and education on the quantitative and qualitative aspects of semantic verbal fluency. Journal of Clinical and Experimental Neuropsychology, 26, 266-277. doi: 10.1076/jcen.26.2.266.28089

Folia, V. \& Kosmidis, M.H. (2003). Assessment of memory skills in illiterates: strategy differences or test artifact? The Clinical Neuropsychologist, 17, 143-152. doi: 10.1076/clin.17.2.143. 16505

Franzen, S., Van den Berg, E., Goudsmit, M., Jurgens, C., Van de Wiel, L., Kalkisim, Y., ... Papma, J. (2020). A systematic review of neuropsychological tests for the assessment of dementia in non-Western, low-educated or illiterate populations. Journal of the International Neuropsychological Society, 26, 331-351. doi: 10.1017/S1355617719000894

Ganguli, M., Ratcliff, G., Chandra, V., Sharma, S., Gilby, J., Pandav, R., ... Dekosky, S. (1995). A Hindi version of the MMSE: the development of a cognitive screening instrument for a largely illiterate rural elderly population in India. International Journal of Geriatric Psychiatry, 10, 367-377. doi: 10.1002/gps.930100505

Gómez, F., Zunzunegui, M.V., Lord, C., Alvarado, B., \& García, A. (2013). Applicability of the MoCA-S test in populations with little education in Colombia. International Journal of Geriatric Psychiatry, 28, 813-820. doi: 10.1002/gps.3885.

Goudsmit, M., Uysal-Bozkir, Ö., Parlevliet, J.L., van Campen, J.P., de Rooij, S.E., Schmand, B. (2017). The Cross-cultural dementia screening (CCD): a new neuropsychological screening instrument 
for dementia in elderly immigrants. Journal of Clinical and Experimental Neuropsychology, 39, 163-172. doi: 10.1080/ 13803395.2016.1209464

Goudsmit, M., van Campen, J.P.C.M., Franzen, S., van den Berg, E., Schilt, T., \& Schmand, B. (2020). Dementia detection with a combination of informant-based and performance-based measures in low-educated and illiterate elderly migrants. Clinical Neuropsychologist. doi: 10.1080/13854046.2020.1711967

Hong, Y.J., Yoon, B., Shim, Y.S., Cho, A.H., Lee, E.S., Kim, Y.I., \& Yang, D.W. (2011). Effect of literacy and education on the visuoconstructional ability of non-demented elderly individuals. Journal of the International Neuropsychological Society, 17, 934-939. doi: 10.1017/S1355617711000889

Javadi, P.S., Zendehbad, A., Darabi, F., Khosravifar, S., \& Noroozian, M. (2015). Development and implementation of Persian test of Elderly for Assessment of Cognition and Executive function (PEACE). Electronic Physician, 7, 1549-1556. doi: 10.19082/1549

Jovanovski, D., Zakzanis, K., Ruttan, L., Campbell, Z., Erb, S., \& Nussbaum, D. (2012). Ecologically valid assessment of executive dysfunction using a novel virtual reality task in patients with acquired brain injury. Applied Neuropsychology-Adult, 19, 207-220. doi: 10.1080/09084282.2011.643956

Kang, S.G., Cho, S.J., Ryu, S.H., Choi, S.H., Han, S.H., Shim, Y.S., ... Lee, D.W. (2015). Normative study of the literacy independent cognitive assessment in illiterate and literate elderly Koreans. Psychiatry Investigation, 12, 305-315. doi: 10.4306/pi. 2015.12.3.305

Kempler, D., Teng, E.L., Taussig, M., \& Dick, M.B. (2010). The common objects memory test (COMT): a simple test with cross-cultural applicability. Journal of the International Neuropsychological Society, 16, 537-545. doi: 10.1017/s135561771 0000160

Kim, H.J., Baek, M.J., \& Kim, S. (2014). Alternative type of the Trail Making test in nonnative English-speakers: the Trail Making Test-black \& white. PLoS One, 9. doi: 10.1371/journal. pone. 0089078

Kim, H. \& Chey, J. (2010). Effects of education, literacy, and dementia on the Clock Drawing Test performance. Journal of the International Neuropsychological Society, 16, 1138-1146. doi: 10.1017/S1355617710000731. Epub 2010 Oct 20.

Kosmidis, M.H. (2018). Challenges in the neuropsychological assessment of illiterate older adults. Language, Cognition and Neuroscience, 33, 373-386. doi: 10.1080/23273798.2017.13 79605

Kosmidis, M.H., Tsapkini, K., \& Folia, V. (2006). Lexical processing in illiteracy: effect of literacy or education? Cortex, 42, 1021-1027. doi: 10.1016/S0010-9452(08)70208-9

Kosmidis, M.H., Vlahou, C.H., Panagiotaki, P., \& Kiosseoglou, G. (2004). The verbal fluency task in the Greek population: normative data, and clustering and switching strategies. Journal of the International Neuropsychological Society, 10, 164-172. doi: 10. 1017/S1355617704102014

Kosmidis, M. H., Zafiri, M., \& Politimou, N. (2011). Literacy versus formal schooling: influence on working memory. Archives of Clinical Neuropsychology, 26, 575-582. doi: 10.1093/arclin/ acr063

Lachmann, T., Khera, G., Srinivasan, N., \& van Leeuwen, C. (2012). Learning to read aligns visual analytical skills with grapheme-phoneme mapping: Evidence from illiterates. Frontiers in Evolutionary Neuroscience, 4. doi: 10.3389/fnevo.2012.00008

Landgraf, S., Beyer, R., Schaadt, G., \& van der Meer, E. (2011). Dissociating improvement of attention and intelligence during written language acquisition in adults. International Journal of Intelligence Science, 1, 17-24. doi: 10.4236/ijis.2011.12003

Lawton, M.P. \& Brody, E.M. (1969). Assessment of older people: self-maintaining and instrumental activities of daily living. The Gerontologist, 9, 179-186. doi: 10.1093/geront/9.3_Part_1.179

Lezak, D.M., Howieson, D.B., \& Loring, D.W. (2004). Neuropsychological Assessment (4th Ed). New York: Oxford University Press.

Maillet, D., Matharan, F., Clésiau, H.L., Bailon, O., Pérès, K., Amieva, H., Belin, C. (2016). TNI-93: a new memory test for dementia detection in illiterate and low-educated patients. Archives of Clinical Neuropsychology, 31, 896-903. doi: 10. 1093/arclin/acw065

Manly, J.J., Jacobs, D.M., Sano, M., Bell, K., Merchant, C.A., Small, S.A., \& Stern, Y. (1999). Effect of literacy on neuropsychological test performance in nondemented, education-matched elders. Journal of the International Neuropsychological Society, 5, 191-202.

Morris, J.C. (1993). The Clinical dementia rating (CDR). Neurology, 43, 2412 LP-2412-a. Retrieved from http://n. neurology.org/content/43/11/2412.2.abstract

Nell, V. (2000). Cross-cultural Neuropsychological Assessment: Theory and Practice. Mahwah, NJ: Lawrence Erlbaum Associates.

Nielsen, T.R. \& Jørgensen, K. (2013). Visuoconstructional abilities in cognitively healthy illiterate Turkish immigrants: a quantitative and qualitative investigation. Clinical Neuropsychologist, 27, 681-692. doi: 10.1080/13854046.2013.767379

Nielsen, T.R., Phung, T.K., Chaaya, M., Mackinnon, A., Waldemar, G. (2016). Combining the Rowland Universal Dementia Assessment Scale and the Informant Questionnaire on Cognitive Decline in the elderly to improve detection of dementia in an Arabic-speaking population. Dementia and Geriatric Cognitive Disorders, 41, 46-54. doi: 10.1159/000 441649.

Nielsen, T.R., Vogel, A., \& Waldemar, G. (2012). Comparison of performance on three neuropsychological tests in healthy Turkish immigrants and Danish elderly. International Psychogeriatrics, 24, 1515-1521. doi: 10.1017/s1041610212 000440.

Nielsen, T.R. \& Waldemar, G. (2016). Effects of literacy on semantic verbal fluency in an immigrant population. Neuropsychology, Development, and Cognition. Section B, Aging, Neuropsychology and Cognition, 23, 578-590. doi: 10.1080/13825585.2015. 1132668

Nitrini, R., Caramelli, P., Herrera Júnior J., Porto, C.S., Charchat-Fichman, H., Carthery, M.T., ... Lima, E.P. (2004). Performance of illiterate and literate nondemented elderly subjects in two tests of long-term memory. Journal of the International Neuropsychological Society, 10, 634-638. doi: 10.1017/S1355617704104062

Ortega, L.F.V., Aprahamian, I., Borges, M.K., Cação, J.C., \& Yassuda, M.S. (2019). Screening for Alzheimer's disease in low-educated or illiterate older adults in Brazil: a systematic review. Arq Neuropsiquiatr, 77, 279-288. doi: 10.1590/0004282X20190024

Ostrosky-Solis, F., Ardila, A., \& Rosselli, M. (1999). NEUROPSI: a brief neuropsychological test battery in Spanish with norms by age and educational level. Journal of the International Neuropsychological Society, 5, 413-433.

Paddick, S.M., Gray, W.K., McGuire, J., Richardson, J., Dotchin, C., Walker, R.W. (2017). Cognitive screening tools 
for identification of dementia in illiterate and low-educated older adults, a systematic review and meta-analysis. International Psychogeriatrics, 29, 897-929. doi: 10.1017/s1041610216001976

Reis, A., \& Castro-Caldas, A. (1997). Illiteracy: a cause for biased cognitive development. Journal of the International Neuropsychological Society, 3, 444-450.

Reis, A., Faisca, L., Mendoca, S., Ingvar, M., \& Petersson, K.M. (2007). Semantic interference on a phonological task in illiterate subjects. Scandinavian Journal of Psychology, 48, 69-74. doi: 10.1111/j.1467-9450.2006.00544.x

Reis, A., Guerreiro, M., \& Petersson, K.M. (2003). A sociodemographic and neuropsychological characterization of an illiterate population. Applied Neuropsychology, 10, 191-204. doi: 10. 1207/s15324826an1004_1

Reis, A., Petersson, K.M., Castro-Caldas, A., \& Ingvar, M. (2001). Formal schooling influences two- but not three-dimensional naming skills. Brain and Cognition, 47, 397-411. doi: 10. 1006/brcg.2001.1316

Salmon, D.P., Jin, H., Zhang, M.Y., Grant, I., \& Yu, E. (1995). Neuropsychological assessment of Chinese elderly in the Shanghai Dementia Survey. The Clinical Neuropsychologist, 9, 159-168. doi: 10.1080/13854049508401598

Thompson, T.A.C., Wilson, P.H., Snyder, P.J., Pietrzak, R.H., Darby, D., Maruff, P., \& Buschke, H. (2011). Sensitivity and test-retest reliability of the International Shopping List Test in assessing verbal learning and memory in mild Alzheimer's disease. Archives of Clinical Neuropsychology, 26, 412-424. doi: 10.1093/arclin/acr039

Tiwari, S.C., Tripathi, R.K., \& Kumar, A. (2009). Applicability of the Mini-mental State Examination (MMSE) and the Hindi Mental State Examination (HMSE) to the urban elderly in India: a pilot study. International Psychogeriatrics, 21: 123-128. doi: 10.1017/S1041610208007916.

Tsapkini, K., Vlahou, C.H., \& Potagas, C. (2010). Adaptation and validation of standardized aphasia tests in different languages: lessons from the Boston Diagnostic Aphasia Examination Short Form in Greek. Behavioural Neurology, 22, 111-119. doi: 10.3233/ben-2009-0256
UNESCO eAtlas of Literacy. Accessed July 30, 2020 at https:// tellmaps.com/uis/literacy/\#!/tellmap/1362426396

van Linden, S. \& Cremers, A.H.M. (2008). Cognitive abilities of functionally illiterate persons relevant to ICT use. In K. Miesenberger, J. Klaus, W. Zagler, \& A. Karshmer (Eds.), Computers helping people with special needs, (pp. 705-712). Heidelberg, Germany: Springer-Verlag.

Vlahou, C.H. \& Kosmidis, M. (2002). The Greek Trail making test: preliminary normative data for clinical and research use. Psychologia: The Journal of the Hellenic Psychological Society, 9, 336-352.

Vlahou, C.H., Kosmidis, M.H., Dardagani, A., Tsotsi, S., Giannakou, M., Giazkoulidou, A., ... Pontikakis, N. (2013). Development of the Greek verbal learning test: reliability, construct validity, and normative standards. Archives of Clinical Neuropsychology, 28, 52-64. doi: 10.1093/arclin/acs099

Wilson, B., Cockburn, J., Baddeley, A., \& Hiorns, R. (1989). The development and validation of a test battery for detecting and monitoring everyday memory problems. Journal of Clinical and Experimental Neuropsychology, 11, 855-870. doi: 10.1080/ 01688638908400940

Wright, M.J., Woo, E., Schmitter-Edgecombe, M., Hinkin, C.H., Miller, E.N., \& Gooding, A.L. (2009). The item-specific deficit approach to evaluating verbal memory dysfunction: rationale, psychometrics, and application. Journal of Clinical and Experimental Neuropsychology, 31, 790-802. doi: 10.1080/ 13803390802508918

Yassuda, M.S., Diniz, B.S.O., Flaks, M.K., Viola, L.F., Pereira, F.S., Nunes, P.V, \& Forlenza, O.V. (2009). Neuropsychological profile of Brazilian older adults with heterogeneous educational backgrounds. Archives of Clinical Neuropsychology, 24, 71-79. doi: 10.1093/arclin/acp009

Youn, J.H., Siksou, M., Mackin, R.S., Choi, J.S., Chey, J., \& Lee, J.Y. (2011). Differentiating illiteracy from Alzheimer's disease by using neuropsychological assessments. International Psychogeriatrics, 23, 1560-1568. doi: 10.1017/S1041610211 001347 